\title{
On the value of test data for reducing uncertainty in material models: Computational framework and application to spherical indentation
}

\author{
Erfan Asaadi ${ }^{\mathrm{a}, \mathrm{b}, *}$, P. Stephan Heyns ${ }^{\mathrm{b}}$, Raphael T. Haftka ${ }^{\mathrm{c}}$, Mazdak Tootkaboni ${ }^{\mathrm{a}}$ \\ ${ }^{\text {a }}$ Department of Civil and Environmental Engineering, University of Massachusetts Dartmouth, Dartmouth, MA, United States \\ ${ }^{\mathrm{b}}$ Centre for Asset Integrity Management, Department of Mechanical and Aeronautical Engineering, University of Pretoria, Pretoria, South \\ Africa ${ }^{\mathrm{c}}$ Department of Mechanical \& Aerospace Engineering, University of Florida, Gainesville, FL, United States
}

\footnotetext{
* Corresponding author at: Department of Civil and Environmental Engineering, University of Massachusetts Dartmouth, Dartmouth, MA, United States.E-mail address: er.asaadi@gmail.com (E. Asaadi).
}

\begin{abstract}
We present a conceptual framework and the computational tools to study the value of the material responses in designing material characterization tests to identify the material model under uncertainty. A computational framework is first developed to estimate the information gained by observing a material response as a measure of the value of the experiment. The proposed framework is then extended to estimate the mutual information between the material response space and the material model space as a basis for ranking the available material response candidates as they relate to reducing the uncertainty of the inferred model. We then define a design problem where a tunable parameter, referred to as the design parameter, is identified so as to render two different material responses to be of the same value from an information content point of view. We finally study the value of the material responses, obtained in a spherical indentation test, i.e. reaction force-indenter displacement, maximum indentation load and the residual imprint, where it is shown that the proposed framework offers a computationally affordable and uncertainty-aware platform to design material characterization tests.
\end{abstract}

Keywords: Uncertainty analysis; Information gain; Mutual information; Indentation test; Material model identification; Design of experiment

\section{Introduction}

The goal of inverse analysis is to infer the non-measurable parameters of a system from its measurable responses. In material model identification, inverse problems are defined to infer the material model parameters from the material response. Conducting a material test, a particular response $D$ from the material response space $\boldsymbol{D}$ is measured. The aim of the inverse analysis is then to identify the material parameter values $\theta$ belonging to the parameter space $\Theta$, for which the simulated response best agrees with the material response. The literature is rife with application of the deterministic inverse analysis methods in constitutive model identification (see e.g. [1] and references therein). These conventional methods estimate the material parameters by solving an optimization problem [2-7], or through constructing an inverse map, (see e.g. [8,9]). A more informative, yet demanding approach to solve an inverse problem 
is to take the influence of the uncertainty sources into account. This is done through devising a probabilistic inverse problem for which the solution provides the uncertainty of the identified model parameters as well [10-16]. In the probabilistic formulation of the material identification problem, the material parameters are not point estimates; rather they are random variables described by their probability distributions.

A close look at attempts aimed at formulating and solving probabilistic material model identification problems (see e.g. [10-15]) reveals the absence of a formal strategy for measuring the value of different material responses. The important question to ask is: from the set of candidate material responses, $\boldsymbol{D}=\left\{D_{1}, D_{2}, \ldots, D_{n}\right\}$, which response is more informative for our inference purposes? In other words, how can we select the proper material response and tune the test parameters to increase the information content of the outcome of the experiment, and reduce the uncertainty of the inferred model. In the context of material model inference this would mean reducing the uncertainty from prior state (when the material response is not available) to posterior state, when we have performed the experiment and obtained the material response.

Several studies in material model identification literature have studied the value of the material response candidates in a deterministic way [17-19]. For instance, in the study conducted in [19], the weighted sum of residual imprint responses obtained in an indentation test was employed to form the material response to identify the hardening parameters. The weights, which represented the value of each response, were tuned such that the material parameters, identified in eight random restarts of the optimization algorithm, converged to the same value and approached the parameters identified in a tensile test of the studied material. While these works have made an attempt to bring in the value of material response into the identification process, an objective measure of the value of the response is still missing. A quantitative indicator for ranking different mechanical responses of materials based on the deformation heterogeneity, strain level and the state of strain was proposed in [20]. This work, however, overlooked the importance of a probabilistic approach to ranking material responses based on the uncertainty of the inferred material model/parameters.

This article presents a framework for addressing the influence of uncertainty sources and the "value" of the material response on the process of material model identification. The identification approach adopted will be that of Bayesian inference which has received increased traction in recent years [21-24]. We meld Bayesian inverse analysis, information theory, Finite Element simulation, surrogate modeling, and efficient sampling to develop a framework for uncertainty-informed material model identification. The value of material response is measured based on concepts such as information gain and mutual information, and computational difficulties associated with the evaluation of model parameters' posterior distributions and model evidence are alleviated via the adaptation of nested sampling [25]. The specific design of the proposed framework facilitates the examination of the material response candidates in the process of material model identification. We represent the application of our proposed computational framework to rank not only the different responses of an indentation test, but also the different response spaces. This contrasts with the current state of practice where study of the indentation test responses is done deterministically and limited to ranking the known material responses at a limited number of observations [17-19]. Nevertheless, we note here that the choice of the spherical indentation test in this work is only for the sake of illustration and the approaches and tools that comprise the proposed framework are applicable to other material characterization tests.

The organization of the paper is as follows. We provide the ingredients of our proposed information-driven framework in Section 2. This includes a brief overview of the basics of Bayesian model calibration, which could be skipped if the reader is already familiar with these concepts, and some preliminary thoughts on different strategies for ranking material responses and their advantages and disadvantages (Section 2.1). It also includes an overview of some of the key concepts in information theory such as entropy, information gain and mutual information [26,27] (Section 2.2.1), followed by the details of the computational strategies that allow for the quantification of the value of the material responses in an affordable way (Section 2.2.2). In Section 3, we apply the proposed framework to study the value of material responses in an indentation test, i.e. residual imprint (RI), maximum indentation load (MIL), and the reaction force-indenter displacement (RFID), and to identify the parameters of a power law material hardening model. Specifically, after providing the details that pertain to the construction of the forward model and likelihood function (Sections 3.2 and 3.3), we estimate the information gain for different choices of material response, and the mutual information between the material response space and the material model parameter space (Section 3.4). Aside from laying down an information-theoretic path to selecting proper material responses for model parameter inference, Section 3 also provides some insight on how to adjust the (tunable) design parameters of an experiment based on the knowledge about the uncertainty sources, before conducting the experiment. Concluding remarks are provided in Section 4. 


\section{Information-driven material model characterization}

\subsection{Bayesian model identification and the ranking of material responses}

In model calibration through Bayesian inference, the model parameters are assumed to follow probability distributions, often reflecting the prior information about them, rather than taking on fixed and certain values. The prior belief about the parameters is then updated based on the information obtained from the experiments to form the posterior distribution of the model parameters. This can be summarized in the form of Bayes' rule as

$$
p(\theta \mid D, \boldsymbol{D})=\frac{p(D \mid \theta, \boldsymbol{D}) \times p(\theta)}{p(D \mid \boldsymbol{D})},
$$

in which $\mathcal{L}(\theta)=p(D \mid \theta, \boldsymbol{D})$ is the likelihood function, representing the likelihood of observing $D$ given the model parameters $\theta,[13,28]$. For a known response $D, \mathcal{L}(\theta)$ represents the likelihood as the function of model parameters, $p(\theta)$ is the prior distribution of the parameters, and represents our prior belief about the parameters, and $p(\theta \mid D, \boldsymbol{D})$ is the posterior distribution of the model parameters given the data. Finally, $p(D \mid \boldsymbol{D})$ is marginalized likelihood, or the model evidence, which is defined as

$$
p(D \mid \boldsymbol{D})=\int p(D \mid \theta, \boldsymbol{D}) \times p(\theta) d \theta .
$$

Needless to say, to infer the model parameters from the system response, the response must be correlated with the parameters of interest. In other words, the response must contain sufficient information about the sought-after parameters. The Bayesian inverse modeling relies on the expectation that after observing the material response, the posterior knowledge, described by $p(\theta \mid D, D)$, reduces the initial uncertainty about the parameters, described by $p(\theta)$; see $[10,28]$ for more details.

Acknowledging the uncertainty of the model parameters, any utility function (objective function), which is employed to rank the material responses should be a function of the posterior distribution of the model parameters. For instance, if the aim of the test is identifying the posterior precision of all the model parameters, the inverse of the determinant of the posterior covariance matrix could serve as the utility function. This is known as "Bayesian D-posterior precision"; e.g. see [29,30]. However, if one is interested in the precision of the marginal posterior distributions of the model parameters, the trace of the posterior covariance matrix, instead of its determinant, can be used to obtain the "Bayesian A-posterior precision".

On the other hand, if the posterior distribution is multi-modal, using a variance based objective such as "Bayesian D-posterior precision" may be inappropriate [31] as the ranking will be affected by not only the scatter of the data around each mode (representing the uncertainty), but also by the distance between the modes. To avoid such scenarios, it is suggested that information gain, in which uncertainty is measured as entropy, and mutual information between prior and posterior distributions be used; see [21,22,24,31] for recent applications of these concepts in design of experiments. It is, however, noted that both variance and entropy are measures of uncertainty, and (from a computational point of view) either of variance-based and entropy-based utility functions would require the exploration of the posterior distributions. It is also noted that of utmost importance is the ability to rank not only the observations (given responses) in an inference problem, but also the space of the responses that the observations belong to. This further, justifies using the mutual information, a measure of dependency, between the response space and the parameter space as a utility function which incorporates the value of all possible observations from a certain response space in an average sense. The ranking of the response spaces, using this measure, then guides the decision maker to choose the response class, from among the response class candidates, for which the possible observations are expected to reduce the uncertainty of the inferred model the most. In what follows we lay out the details of a framework where concepts from information theory are used to measure the value of material responses in a computationally affordable way.

\subsection{An information-theoretic view of the value of material response}

The word information is frequently used in the material response selection and the design of material tests. Throughout this manuscript, we make use of the language of information theory, [26,27], in which information and entropy are defined and quantified for a realization of the random variable and the random variable itself respectively. 
The idea is to measure the information content of the material responses and use it to design experiments that allow for inferring the parameters of interest in a material model in such a way as to reduce the uncertainty of the inferred parameters. We note that the ultimate goal often is to predict a particular response not examined through experiments, e.g. the response of the material in a forming process. Considering the fact that models are only idealizations of the reality, a material model which best describes the test response, does not necessarily predict the response of the material the best under a different test condition. In this study, however, we set our goal to infer the model parameters from a particular observed material response implicitly relying on the premise that the identified model is to be used in the neighborhood of the test condition.

\subsubsection{Information gain and mutual information as measures of value}

In information theory, the entropy of a continuous random variable (or vector) $\theta \sim p(\theta)$ is defined by

$$
\mathrm{H}(\boldsymbol{\Theta})=-\int p(\theta) \log (p(\theta)) d \theta=-\mathbb{E}_{\theta}(\log (p(\theta))),
$$

where $\mathbb{E}_{\theta}$ represents the expected value operator and $p(\theta)$ represents the prior (either joint or marginal) distribution of the parameters [26]. Lindley [27] was perhaps the first to take an information theory approach to compare experiments. Lindley's work was unique in the sense that prior work in the area of probabilistic design of experiments had mainly focused on the effect of choice of experiment on the risk associated with a particular decision and not on the value of response measured, see [32]. Defining the amount of information in the prior distribution of the model parameters, to be the negative of the entropy, an estimate of the information content (for a particular choice of prior) can be obtained using Monte Carlo (MC) simulation:

$$
g_{0}=-\mathrm{H}(\boldsymbol{\Theta})=\frac{1}{N} \sum_{\theta \sim p(\theta)} \log (p(\theta)),
$$

in which $\theta$ is drawn from $p(\theta)$ and $\mathrm{N}$ is the number of MC samples.

Observing the material response $D$, the posterior distribution is obtained and the conditional entropy is defined as

$$
\mathrm{H}(\boldsymbol{\Theta} \mid \mathbf{D}=D)=-g_{1}(D)=-\int p(\theta \mid D) \log (p(\theta \mid D)) d \theta
$$

Lindley [27] defined $g_{1}(D)$ as the amount of information about $\Theta$, after observing the response $D$. On average, however, the conditional entropy between the parameter space $\Theta$ and the response space $\mathbf{D}$ is

$$
\mathrm{H}(\boldsymbol{\Theta} \mid \mathbf{D})=-\int p(D) \mathrm{H}(\boldsymbol{\Theta} \mid \mathbf{D}=D) d D=-\int p(D) \int p(\theta \mid D) \log (p(\theta \mid D)) d \theta d D
$$

for which the MC estimate would be

$$
\mathrm{H}(\boldsymbol{\Theta} \mid \mathbf{D})=-\frac{1}{N} \sum_{\theta, D \sim p(\theta, D)} \log (p(\theta \mid D)) .
$$

We note that, $\theta$ and $D$ are samples drawn from the joint distribution $p(\theta, D)$. The mutual information between $\Theta$ and $\mathbf{D}$, the parameter space and the response space, is then defined as

$$
\mathrm{I}(\boldsymbol{\Theta}, \mathbf{D})=\mathrm{H}(\boldsymbol{\Theta})-\mathrm{H}(\boldsymbol{\Theta} \mid \mathbf{D}),
$$

which is symmetric, that is $\mathrm{I}(\boldsymbol{\Theta}, \mathbf{D})=\mathrm{I}(\mathbf{D}, \boldsymbol{\Theta})$, and represents how much, on average, the realization of $\mathbf{D}$, tells about the realization of $\Theta$.

Considering Bayes' rule in Eq. (1), we can rewrite Eq. (8) as

$$
\mathrm{I}(\boldsymbol{\Theta}, \mathbf{D})=\int_{\theta, D} p(\theta, D) \log \left(\frac{p(D \mid \theta)}{p(D)}\right) d \theta d D,
$$

The primary step in the design of a material test is to select the 'proper' material response, the response containing sufficient amount of information for the inference problem in hand. Assuming we know the measurement uncertainty in the form of measurement noise inherent in different measurements, the goal is to identify "what measurement to use" to reduce the uncertainty of the inferred model. To this end, we suggest to first estimate the mutual information between the response space and the parameter space before we embark on conducting the test in the process of material 
model identification. Subsequently, the material response with the maximum value of the mutual information between the response and the parameter spaces will be selected. In addition, the experiment may comprise one or more design parameters $x$, which influence the response. To maximize the value of the experiment we then maximize $\mathrm{I}(\Theta, \mathbf{D}(\mathrm{x}))$ to identify the optimum design parameters as

$$
x_{\text {opt }}=\operatorname{argmax}(\mathrm{I}(\boldsymbol{\Theta}, \mathbf{D}(x))) .
$$

Alternatively, $x_{\text {opt }}$ can be selected to identify the alternative material responses, the responses with the same amount of information. The objective function is then defined as $\left(\mathrm{I}\left(\Theta, \mathbf{D}_{1}(\mathrm{x})\right)-\mathrm{I}\left(\Theta, \mathbf{D}_{2}(\mathrm{x})\right)\right)^{2}$, minimization of which means material responses $\mathbf{D}_{1}(\mathrm{x})$ and $\mathbf{D}_{2}(\mathrm{x})$ contain as close as an amount of information as possible; ideally equal amounts of information $\left(\mathrm{I}\left(\Theta, \mathbf{D}_{1}(\mathrm{x})\right)=\mathrm{I}\left(\Theta, \mathbf{D}_{2}(\mathrm{x})\right)\right)$ if the range of admissible values for $x_{\text {opt }}$ allows reaching zero. This could be, in particular, useful when two responses are measured differently and different uncertainty sources are involved in each measurement. Identifying an alternative test, one can select the test method based on other factors, e.g. cost, rather than the information gained by conducting the test. We note that, in the general case, in which the amount of information is not the only objective, one may face a multi-objective optimization problem, and selecting the design variable may comprise a trade-off between different objectives. We also note that, in this paper, our emphasis is less on searching for the optimal design parameter from the multidimensional design parameter space, commonly known as design of experiment (see the review in [31]) and more on providing insight into the value of the material responses by estimating the mutual information and entropy for known design parameters. In Section 3.4, however, we make an attempt to identify the magnitude of the uncertainty of the machine compliance, our design parameter in the indentation test, in which two indentation responses, i.e. residual imprint + maximum indentation load and reaction force-indenter displacement response contain the same amount of information.

Considering that computing $\mathrm{I}(\boldsymbol{\Theta}, \mathbf{D})$ is not a straightforward task, we make use of MC simulation again $[21,33]$, that is we write

$$
\mathrm{I}(\boldsymbol{\Theta}, \mathbf{D})=\frac{1}{N} \sum_{D_{i}, \theta_{i} \sim p\left(D_{i}, \theta_{i}\right)}\left[\log \left(p\left(D_{i} \mid \theta_{i}\right)\right)-\log \left(p\left(D_{i}\right)\right)\right] .
$$

Eq. (11) comprises two terms. First, $\log \left(p\left(D_{i} \mid \theta_{i}\right)\right)$, which is the likelihood function evaluated at the samples drawn from the joint distribution $p\left(D_{i}, \theta_{i}\right)$, is computed. To this end, we draw $\theta_{i} \sim p\left(\theta_{i}\right)$ and $D_{i}$ from conditional distribution $p\left(D_{i} \mid \theta_{i}\right)$. That is, we simulate the experiment (e.g. the indentation test) at $\theta_{i}$ taking the influence of noise and all uncertainty sources into account. The main computational effort in calculating the mutual information is, however, related to the estimation of the marginalized likelihood, $p\left(D_{i}\right)$. In what follows we lay out the details of an effective strategy that allows for an effective estimation of information and entropy in the context of design of material characterization tests.

\subsubsection{Information quantification: computational aspects}

As it was described, computing the entropy, and subsequently mutual information analytically is often intractable. This is particularly so when the model response is generated using a black-box model such as a Finite Element (FE) model, or a finite difference PDE solver, which is almost always the case in material model identification problems. MC simulation is therefore usually adopted to estimate the integrals in Eq. (11). However, naïve sampling, with a limited number of samples as is usually the case, hinders full exploration of the bulk of the distribution and may result in loss of accuracy. Therefore, a large number of samples is needed to fairly explore the entire support of the distribution. This may make classical MC estimation of entropy and mutual information for the models with more than three parameters impractical, particularly when the likelihood function is highly peaked.

To estimate I $(\boldsymbol{\Theta}, \mathbf{D})$, brute force MC was employed to design the experiments in system biology [24]. Huan and Marzouk [21] used special MC sampling, where samples drawn from the prior distribution were used to generate both terms in Eq. (11). Assuming Gaussian posteriors for the model parameters, Laplace approximation was suggested in $[22,23]$ to approximate the information gain. Other sampling techniques have been adopted to approximate the terms needed in Eq. (10) or Eq. (5), to estimate the mutual information and entropy, see [31]. Pros and cons of various sampling methods to estimate the parameters' posterior distributions and marginalized likelihood are reviewed in [34]. The authors, however, note that the purpose of this paper is not comparing all possible sampling algorithm candidates to estimate the entropy and mutual information. Referring to [34], we opt for Nested Sampling (NS) developed by Skilling [25] to approximately either sample from the posterior, required in the entropy estimation, or estimate the 
marginalized likelihood, required in the mutual information estimation. As it was demonstrated in [10], compared with other sampling algorithms, the advantage of nested sampling is that it allows to estimate the posterior samples and the marginalized likelihood simultaneously, tuning the algorithm's parameters does not involve a cumbersome calibrating process, and the acceptance rate can easily be kept close to 1 (see [34-36]).

The idea of nested sampling is to transform the $\mathrm{N}$-dimensional integral, with $\mathrm{N}$ the dimension of parameter space (vector $\theta$ ) in Eq. (2), to a one dimensional integral. The prior mass, $X$, which represents the fraction of volume in the parameter space with the likelihood greater than $\lambda$ is defined as

$$
X(\lambda)=\int_{P(D \mid \theta, \boldsymbol{D})>\lambda} P(\theta) d \theta^{N} .
$$

With the following transformation we have

$$
p(D)=\int_{0}^{1} \mathcal{L}(X) d X \cong \sum \mathcal{L}_{i} \underbrace{\frac{\left(X_{i+1}-X_{i-1}\right)}{2}}_{w_{i}} .
$$

As $\lambda$ increases, the prior mass decreases from 1 to 0 . Calculating the one-dimensional integral above is not so difficult. However, computing the prior mass $X$ under the condition $\mathcal{L}(\theta)>\lambda$ is not a straightforward task. The computational burden, however, will be alleviated using nested sampling which is summarized as follows (see [25]):

1. L samples from the prior distribution, which is often simply the uniform distribution over the prior range of the parameters, are drawn. This set of samples is known as the live points set.

2. The likelihood of each live point is computed.

3. The algorithm discards the live point with the lowest likelihood $\lambda_{0}$

4. A new sample from the prior space is randomly selected with the constraint $\mathcal{L}\left(\theta_{\text {new }}\right)>\lambda_{0}$, and takes the place of the discarded point.

5. Steps 2-4 are repeated $\mathrm{N}$ times. The discarded points with the order of $\lambda_{0}<\lambda_{1}<\lambda_{2} \ldots<\lambda_{N}$ in each iteration are stored.

Following the steps above, we have a series of samples with the likelihood ordered in an ascending manner. The next step to calculate the transformed integral in Eq. (13) is to compute the corresponding prior mass, which cannot be obtained directly. It can be shown (see [25]) that if we define $t_{i}$ as the largest of L random numbers drawn from the uniform distribution $u$ [01], the random $X_{i}$ and the mean value of prior mass $X_{i}$ is obtained from Eq. (14) as:

$$
X_{i} \cong \exp \left(-\frac{i}{L}\right) \text { (mean value) }, \quad X_{i}=t_{i} X_{i-1} \text { (uncertain) }
$$

The posterior samples are the samples drawn from the prior weighted by the likelihood. Alternatively, these samples can randomly be drawn from the area under $\mathcal{L}-X$. In other words, the discarded points from the nested sampling iterations, weighed as $p_{i}=\mathcal{L}_{i} w_{i} / p(D)$ are the posterior samples.

Drawing a sample from the parameter space with the constraint on the likelihood, $\mathcal{L}(\theta)>\lambda$, is not a straightforward task. Moreover, growing $\lambda$ in each iteration of the sampling results in a steady decrease in the acceptance rate. To improve the acceptance rate, we use ellipsoid sampling [37] to restrict the prior around the live point set in the following manner: a hyperellipse which just touches the maximum coordinate values of the live point set was created and expanded with a small enlargement factor. The live point candidate is then selected randomly within the hyperellipse until the criterion in step 4 is met. In case of significant multimodality, multi ellipsoids or multi clustering (see $[35,36,38]$ ) may be used to isolate the modes and to further improve the acceptance rate.

When the model for the likelihood is in exponential form, arising from the assumption of Gaussianity for the noise, the likelihood values of the samples which are relatively far from the maximum likelihood are assumed zero. However, the total effect of ignoring many small values is not negligible. We, therefore, construct our algorithm based on the logarithmically scaled likelihood values of the samples, generated in each iteration of nested sampling, NS, to avoid this issue. Fortunately, NS is fairly compatible with the logarithmic conversion of the likelihood values. Firstly, if the likelihood is evaluated in the logarithmic scale, the discarded points in the original non-logarithmic scale are still ordered in an ascending manner. Moreover, the log-likelihood of the samples obtained in NS iteration can be easily used to estimate the entropy as

$$
\begin{aligned}
& W_{i}=w_{i} \times \exp \left(\text { loglikelihood }_{i}-Z\right) \\
& p_{i}=\text { loglikelihood }_{i}-Z \\
& \text { Entropy }=-\sum\left(W_{i} \times p_{i}\right) .
\end{aligned}
$$


To estimate the mutual information, we use the already estimated log of marginal likelihood, which is exactly what we need in Eq. (11).

With regard to the performance of Nested sampling in marginalizing high dimensional likelihood functions, we note that we have adopted the technique to characterize posterior distributions in models with over eight parameters; see [10]. In addition, Nested sampling is being used (quite widely) in cosmology to deal with likelihood marginalization for very high-dimensional likelihood functions; see e.g. [36,39]. Having said that, we do not claim that the computational cost of our proposed framework for three to four variable models (such as the one used in this study; see Section 3) and thousand variable models is the same. Nevertheless, we emphasize that one should not compare computational cost of estimating mutual information between material model space and material response space with that of model parameter identification for a given material response. Unlike model identification and model selection problems, mutual information estimation comprises several thousand likelihood marginalizations which are usually avoided in only-material model identification using MCMC.

While the application of nested sampling will reduce the number of response evaluations required, the identification process still needs running the forward model, e.g. FE model of the material test, a large number of times. To alleviate the computational burden associated with the forward model, in this manuscript, we replace the FE model of the indentation test with a cheap-to-evaluate surrogate model based on Artificial Neural Network (ANN) trained with Bayesian optimization; see [10] for more details. We, however, note that surrogate model selection is a problem-dependent task and needs to take into account the trade-off between the construction costs, calling time and the accuracy of the prediction.

\section{Material model identification via spherical indentation}

In this section, we apply the computational framework, developed in Section 4 to study the value of the material responses in an indentation test and to perform identification of material parameters in a hardening model. An attempt is made to highlight the value of the information-theoretic approach to material model identification. We characterize the material model and the information content of the different responses while considering various uncertainty sources. We illustrate that performing identification using responses with higher information content results in a material model with less uncertainty. The mutual information between the material model space and the space of different material responses is also estimated. It is concluded that certain combinations of the material responses may significantly boost the value of the experiment, which we refer to as information fusion. For the case where there is uncertainty in the machine compliance as the design variable, we identify the degree of the uncertainty for which two different classes of the material response are equally worthwhile.

\subsection{Indentation test and value of material responses}

Thanks to recent developments in inverse identification methods, indentation tests, which are often used to measure or compare the hardness of materials, are also being employed to identify the mechanical behavior of the materials in a broader sense [17-19,40-51]. While indentation test responses may be used to infer different material models, in this study, we opt for the elastoplastic material model characterization with a focus on the influence of different uncertainty sources on the uncertainty of the inferred hardening material model parameters.

A large body of studies (see e.g. [51-53]) characterized elastoplastic material models from the reaction force-indenter displacement (RFID) response of an indentation test (see Fig. 1). The measurement of the indenter displacement is affected by the compliance of the parts between the indenter and the displacement sensor. Therefore, when the knowledge of the machine compliance and the indenter is not readily available, the residual imprint, is introduced as the material response to identify the elastoplastic models [17-19,54]. The residual imprint response $\mathrm{Y}$ is represented as a function of the spatial coordinate $\mathrm{X}$, e.g. $\mathrm{Y}(\mathrm{X})$, and can be measured by a microscope, laser or mechanical probes.

Recent studies to quantify the value of the material responses obtained from an indentation test [17-19] do not take the influence of the uncertainty sources into account. In a deterministic approach to material model characterization, response A may be considered more informative than response $\mathrm{B}$, for instance, when the model parameters are more sensitive to changes in response A. The decision to render a response more informative than other, however, may be different when one considers the degree of uncertainty which could influence the result of inference using responses A or B. In addition, the material model parameters may be inferred from a weighted combination of responses, 


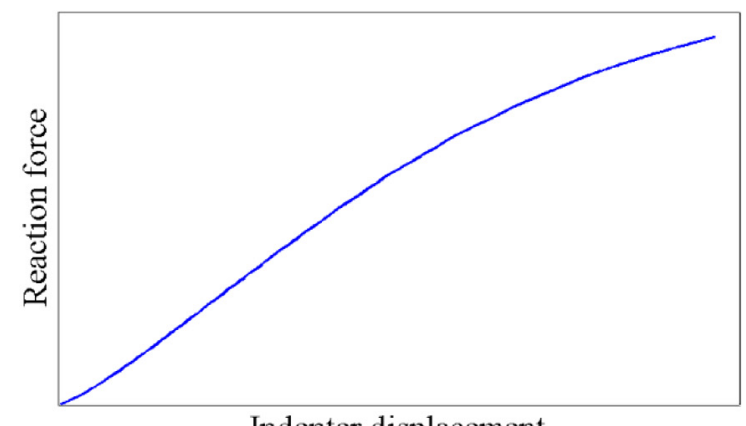

Indenter displacement

(a)

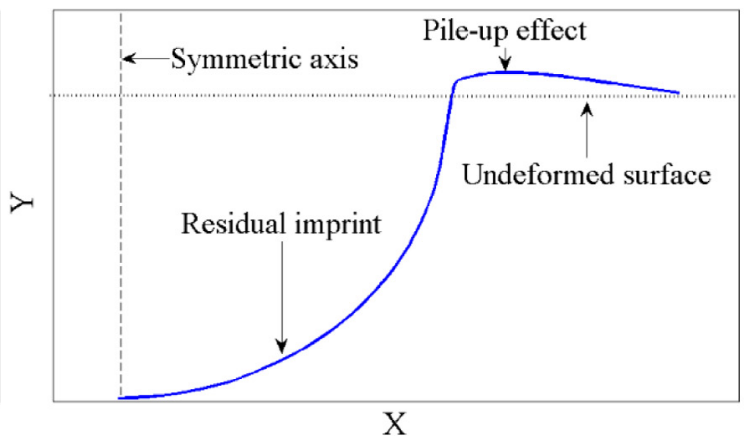

(b)

Fig. 1. Typical material responses in an indentation test. (a) Reaction force-indenter displacement and (b) residual imprint after unloading the indenter.

e.g. a weighted combination of RFID and residual imprint in the case of indentation test $[17,18]$. In such cases the approach to weight and combine responses must consider the degree of uncertainty inherent in each response; otherwise a more uncertain response may receive a higher weight, inappropriately biasing the identification process. Moreover, the decision to favor a specific class of material response over the other as well as how to tune the test's design parameters has to take into account any prior knowledge on the variability or the range of the material model parameters. Therefore, a more global method which compares the response spaces, in an average sense, rather than focusing on a specific response from the response space must be employed.

In what follows, we employ the computational framework developed in Section 2 to provide insight into the choice of material response and its relation to the success of the identification process in the context of spherical indentation and elasto-plastic material model characterization. We emphasize, however, that this particular choice of the test and material response is mainly to lay out the elements of the information theory approach adopted in this work, and to demonstrate how identifying the hardening parameters using responses with low information content, influence the uncertainty of the calibrated model. Through the use of information theory measures, i.e. information gain and mutual information, not only do we estimate the value of the known responses, but also the value of the different response spaces is quantified. Our simulation-based information quantification is a crucial step in conducting the physical experiment in such a way as to reduce the uncertainty of the inferred parameters.

\subsection{Construction of the forward model}

Following the studies conducted in [18,19], the hardening material model used to simulate the material response in this study is chosen as

$$
\sigma<\sigma_{y}: \sigma=\mathrm{E} \varepsilon \quad, \quad \sigma \geq \sigma_{y}: \sigma=\sigma_{y}\left(\frac{E}{\sigma_{y}}\right)^{n} \varepsilon^{n},
$$

in which $\mathrm{E}$ is the Young's modulus, $\sigma_{y}$ is the initial yield and $n$ is the hardening exponent. We make use of two model examples in which the material responses are simulated, considering the measurement uncertainties. Two sets of the material parameters are used to simulate force-indenter depth and the residual imprint responses. The parameters selected to construct Example 1, and Example 2 are given in Table 1 with the second example adopting the material parameters in [19] identified in a uniaxial test for 2099-T83 Al-Li alloys. The distributions of the identified model parameters are then inferred using Bayesian inference and Metropolis-Hasting Markov Chain MHMC sampling. We then estimate the amount of information gained by observing each material response using our computational framework. We conduct our study in three cases: (i) the material response chosen as RFID, (ii) residual imprint as the material response of interest, (iii) the material response chosen as the combination of maximum indentation load and residual imprint.

According to Eq. (1), the likelihood value is the function of the simulated material response. An FE model of the indentation test is used to generate the response from the material test as a function of the material model 
Table 1

Material parameters used to simulate the material response in the indentation test.

\begin{tabular}{llll}
\hline Model example & $\mathrm{E}$ & $\mathrm{n}$ & $\sigma_{y}$ \\
\hline Example 1. & $202(\mathrm{GPa})$ & 0.225 & $300(\mathrm{MPa})$ \\
Example 2. & $77.7(\mathrm{GPa})$ & 0.0678 & $372(\mathrm{MPa})$ \\
\hline
\end{tabular}

Table 2

Inputs and outputs of Artificial Neural Network (ANN) surrogate.

\begin{tabular}{llll}
\hline ANN inputs & & ANN outputs & ANN structure \\
\hline Material parameters & Test parameters & Simulation response & 4.20 .1 \\
\hline$\sigma_{y}, \mathrm{E}, \mathrm{n}$ & Indenter displacement & Reaction force & \\
$\sigma_{y}, \mathrm{E}, \mathrm{n}$ & Maximum indentation depth, residual imprint in x-direction & Residual imprint in y-direction & 5.30 .1 \\
$\sigma_{y}, \mathrm{E}, \mathrm{n}$ & Maximum indentation depth & Maximum indentation load & 4.14 .1 \\
\hline
\end{tabular}

Table 3

Prior ranges of the material model parameters.

\begin{tabular}{llll}
\hline Model example & $\mathrm{E}$ & $\mathrm{n}$ & $\sigma_{y}$ \\
\hline Example 1. & $202-205(\mathrm{GPa})$ & $0.05-0.4$ & $200-400(\mathrm{MPa})$ \\
Example 2. & $75-80(\mathrm{GPa})$ & $0.05-0.4$ & $200-400(\mathrm{MPa})$ \\
\hline
\end{tabular}

parameters. To obtain the maximum indentation depth, we simulate the test for the maximum indenter displacement of $0.165 \mathrm{~mm}-0.175 \mathrm{~mm}$. The maximum indentation depth is then obtained after unloading the indenter. The residual imprint in $x$-direction and $y$-direction are extracted from the coordinates of the surface nodes. As each likelihood evaluation requires an expensive FE simulation of the test, to reduce the computational time we make use of a cheap-to-evaluate surrogate model. Following [10] in this study we employ ANN based surrogate models to generate reaction force and residual imprint responses. To build the surrogate model, the model class is passed through a training process over a sparse set of training points which constitute the inputs and outputs of the FE model of the test. Table 2 summarizes the inputs, the outputs and the structure of the surrogate model where the term "ANN structure" represents the number of nodes in input, hidden and output layers. To mimic the reality of indentation test more closely, we also consider uncertainty in test parameters (see Table 2) and build the surrogate model while varying both material and test parameters. We, however, note that if the test parameters are assumed constant vectors, dimension reduction strategies such as Principal Component Analysis (PCA), can be used to reduce the computational burden and to perform the surrogate construction in a low dimensional space spanned by uncorrelated response variables; see $[10,12]$.

\subsection{Construction of the likelihood function}

In this study, we consider the prior distribution of the material parameters to be uniform (and ranging over intervals given in Table 3) while we quantify the information content of the material responses in model example 1 , model example 2 and the mutual information between the model parameter space and the response space.

The elasticity modulus is often considered more certain than the hardening exponent and initial yield stress for the commonly used alloys and metals. Our emphasis is therefore toward the more uncertain parameters, i.e. hardening exponent and the initial yield stress. We first consider the case where the material response is force-indenter depth. The uncertainty of the force measurement is expressed as a percentage of the actual load, as it is common for the load cells. In this study, we assume $F_{\text {measured }} \sim N\left(\mu=F_{A c t}, \sigma_{F}=0.005 F_{A c t}\right)$ in which $F_{\text {Act }}=F_{\text {sim }}$. However, force response is a function of indenter depth, $x$, which itself is uncertain; see Fig. 2. Therefore, to build the likelihood function, for the case where the model response is reaction force-indenter displacement, we need to take the influence of the uncertainty of the independent variables, all the $x$ 's at which we measure the force, into account. The indenter displacement uncertainty, however, is assumed constant, $x \sim N\left(x_{\text {set }}, \sigma_{x}\right)$ with $\sigma_{x}=10^{-3} \mathrm{~mm}$ and $x_{\text {set }}$ the depth 


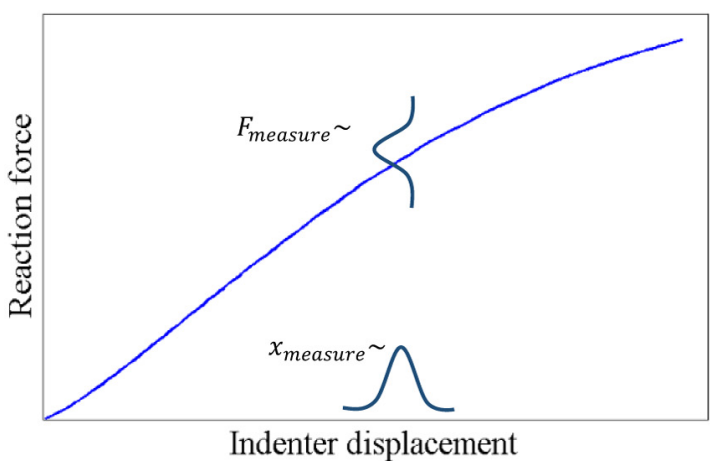

(a)

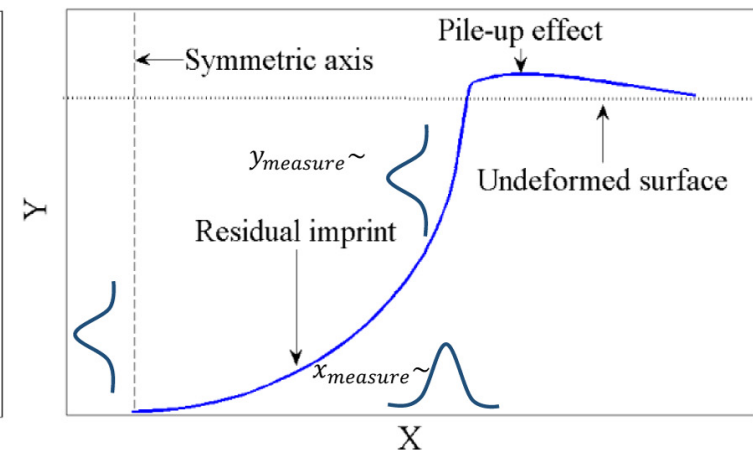

(b)

Fig. 2. Schematic representation of the influence of $x$-uncertainty on the uncertainty of (a) Reaction force, $F(x)$, and (b) Residual imprint, $y(x)$.

at which we "intend" to measure the force. For a single force measurement, at a given $x_{\text {set }}=x_{i}$, the likelihood is expressed as

$$
p_{i}\left(F_{\text {measured }} \mid \theta\right)=\frac{1}{\sqrt{2 \pi \sigma_{F}^{2}}} \exp \left(-\frac{\left(F_{\text {measured }}-F_{\text {sim }}\right)^{2}}{2 \sigma_{F}^{2}}\right)
$$

Knowing the measurement interval, the number of depth observations, $x_{i}$, is known. Writing the likelihood as (17) means we have marginalized with respect to $x$ by averaging over the Monte Carlo samples drawn from $N\left(x_{s e t}, \sigma_{x}\right)$. To reliably perform this marginalization, we need to estimate the number of samples needed to converge at each $x_{i}$. In order to do this, we first estimate the likelihood value at the parameter set which was used to generate the simulated response. We then increase the number of samples (in this study from 300 samples to 500 samples) and check the convergence of the likelihood value.

The measurement interval of the indenter displacement is set at $6 \sigma_{x}$. This interval is usually determined by the resolution of measuring tools. The maximum indenter depth is set at $0.17 \mathrm{~mm}, 0.44 \mathrm{~mm}$ and $0.90 \mathrm{~mm}$ and the information gain and the mutual information are estimated using Eqs. (5) and (11) respectively.

To evaluate the likelihood value for the material parameter set, $\theta$, the inputs of the surrogate model are the parameter set, $\theta$, and vector of the indentation depths, and the output is the vector of the reaction force responses.

When considering the residual imprint as the response of interest, we assume all length measurements have the same uncertainty, $\sigma_{x}, \sigma_{y}=10^{-3} \mathrm{~mm}$. To marginalize the uncertainty of x measurements, the location at which the residual imprint $y$ is measured, we take the same approach as described above. The surrogate model inputs are then the material parameters $\theta$, and the depth of the indentation, which is also uncertain, the vector of $\mathrm{x}$ locations, and the surrogate output is the vector of the vertical imprint values. In the case where we consider the maximum load response to build the likelihood model, the surrogate inputs are the material parameter set and the maximum indentation depth and the output is the force response.

To consider the impact of uncertainty in the machine compliance on the reaction force-indenter displacement response measurements, we model it as a random variable $\alpha$. Therefore, for the measured indenter displacement $\mathrm{x}$, the real displacement is $F(x) / \alpha$ less than $\mathrm{x}$. As $\alpha$ is not of our primary interest, it must be marginalized. To estimate the mutual information in Eq. (11), $p\left(D_{i}\right)$ is estimated using NS, while the model parameters are $\alpha$ and $\theta$. To estimate $\log \left(p\left(D_{i} \mid \theta_{i}\right)\right)$, however, $\alpha$ must be marginalized as

$$
p(D \mid \theta)=\sum_{\alpha \sim p(\alpha)} p(D \mid \theta, \alpha)
$$

The marginalization described in Eq. (18) is against only one variable $\alpha$ over a domain that is a not very large. We therefore use classic MC since it is more convenient than NS when the computational cost is manageable. 
Table 4

Statistical summary of the identified model parameters for the model examples. The material response is force-indenter displacement.

\begin{tabular}{lllllllll}
\hline Examples & Depth & Info. gain & Mean $\sigma_{y}$ & Std. $\sigma_{y}$ & Mean $\mathrm{n}$ & Std. $\mathrm{n}$ & Mean E & Std. E \\
\hline \multirow{3}{*}{ Model example 1 } & 0.17 & 5.24 & 338 & 19.5 & 0.19 & 0.015 & 202 & 1.3 \\
& 0.44 & 7.57 & 293 & 5.89 & 0.23 & 0.004 & 202 & 1.3 \\
& 0.90 & 8.60 & 297 & 2.97 & 0.22 & 0.002 & 202 & 1.3 \\
\hline \multirow{3}{*}{ Model example 2 } & 0.17 & 5.9 & 362 & 9.5 & 0.08 & 0.01 & 78 & 1.4 \\
& 0.44 & 7.92 & 371 & 5.00 & 0.07 & 0.003 & 77 & 1.3 \\
& 0.90 & 8.56 & 370 & 3.93 & 0.07 & 0.002 & 78 \\
\hline
\end{tabular}

Table 5

Statistical summary of the identified model parameters for the model examples. The material response is the imprint and the combination of imprint and maximum indentation load.

\begin{tabular}{lllllllll}
\hline Examples & Depth & Info. gain & Mean $\sigma_{y}$ & Std. $\sigma_{y}$ & Mean n & Std. n & Mean E & Std. E \\
\hline \multirow{2}{*}{ Model example 1 } & Imprint & 4.19 & 277 & 44.9 & 0.229 & 0.005 & 202 & 1.32 \\
& Imprint+ Max load & 6.80 & 298 & 3.4 & 0.23 & 0.002 & 203 & 1.32 \\
\hline \multirow{2}{*}{ Model example 2 } & Imprint & 4.81 & 335 & 31.1 & 0.072 & 0.004 & 78 & 1.33 \\
& Imprint+ Max load & 7.16 & 373 & 2.8 & 0.067 & 0.001 & 78 & 1.31 \\
\hline
\end{tabular}

\subsection{Results and discussion}

\subsubsection{Information gain estimation: reaction force-indenter displacement as the material response}

Fig. 3 represents the distributions of the identified parameters when reaction force-indenter responses are used for Example 1 and Example 2 for the maximum indentation depths of $0.17 \mathrm{~mm}, 0.44 \mathrm{~mm}$ and $0.90 \mathrm{~mm}$. The summary of statistics and the estimated information gain are also represented in Table 4. As can be seen, the amount of information gained by observing the reaction force-indenter displacement response, increases with the extension of the indentation depth. In Example 1, for the maximum indenter depth $0.17 \mathrm{~mm}$, the parameter sets to generate the simulated experiment, the red vertical lines, are at the tail of the identified distribution. This is more or less the same in Example 2. The estimated information gain is also relatively low.

When we increase the maximum indenter depth to $0.44 \mathrm{~mm}$, the uncertainty of the identified parameters shrinks and the expected values of the identified parameter distribution get closer to the parameters used to generate the simulated experiment. Note that, in Example 1, we observe bi-modal distributions for the initial yield and hardening exponent with one of the modes significantly stronger than the other. The non-uniqueness of the parameters identified by the reaction force-indenter displacement response has also been reported in [51,55,56]. As we increase the maximum indenter depth to $0.90 \mathrm{~mm}$ the uncertainty of the initial yield and hardening exponent distributions significantly decreases, which is consistent with the increase in the estimated information gain; see Fig. 3 and Table 4.

\subsubsection{Information gain estimation: residual imprint, and the combination of the residual imprint and maximum indenter load as the response}

We now quantify the information gain by observing the residual imprint response after unloading at the depth of $0.17 \mathrm{~mm}$. This is the minimum indenter depth used to study the information gained by observing the reaction force-indenter displacement response. As illustrated in Fig. 4 and Table 5 the variance of the hardening exponent posterior distributions in Example 1 and Example 2, is lower than that of the distributions obtained when we inferred the parameters from the reaction force-indenter displacement responses at the same indenter depth. However, the variance of the inferred initial yield is much higher. The expected values of initial yield posterior distributions are also relatively far from the yield parameters used to generate the simulated experiment. The estimated information gained by observing the residual imprint response is slightly lower than that associated with observing the reaction force-indenter displacement response at the same depth. It seems the residual imprint response is a good candidate, compared to the reaction force-displacement response, when inferring the hardening exponent, but not when inferring the initial yield value. 

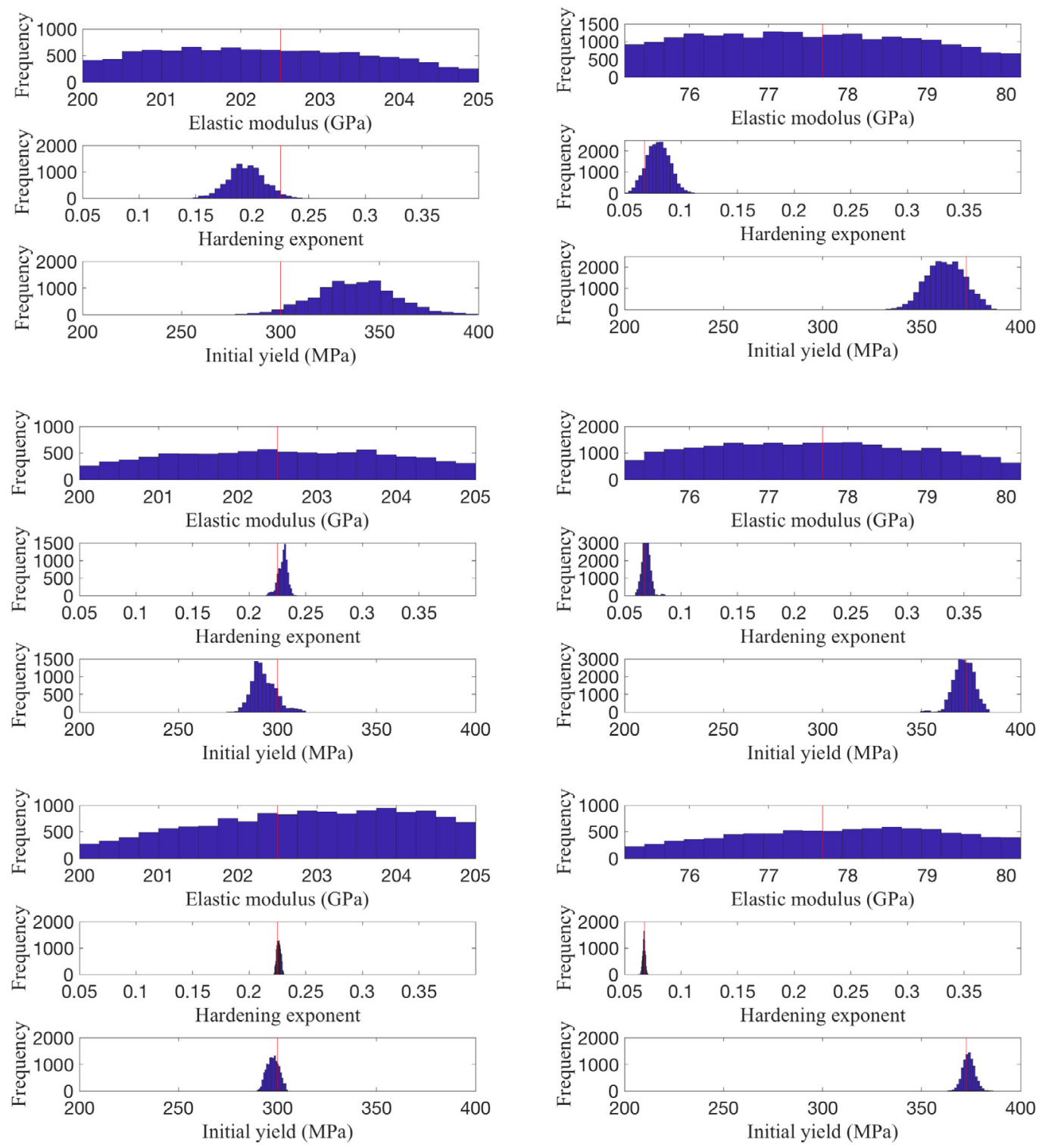

(a)

(b)

Fig. 3. Distributions of the identified parameters for model example 1 on the left and model example 2 on the right. The maximum displacements of the indenter for the three cases (top, middle and bottom) are $0.17 \mathrm{~mm}, 0.44 \mathrm{~mm}$ and $0.90 \mathrm{~mm}$ respectively.

We finally study the information gain while the material response is the residual imprint, vector of $y(x)$, together with the maximum indentation load response, scalar F. As the force and residual imprint are measured independently, the likelihood is the product of the likelihood functions, constructed for each measurement. As depicted in Fig. 4 and summarized in Table 5, considering the maximum indentation load along with the imprint response significantly reduces the uncertainty associated with the identified parameters. The sharp increase in the estimated information gain is also consistent with the reduction of the variance of the posterior distributions. This is known as information fusion.

Needless to say, considering the maximum load response in isolation, the hardening model is significantly unidentifiable. It is worthwhile mentioning that the uncertainty associated with the machine compliance has no effect on the reaction force measurement uncertainty, although it affects the reaction force-indenter displacement measurement. 

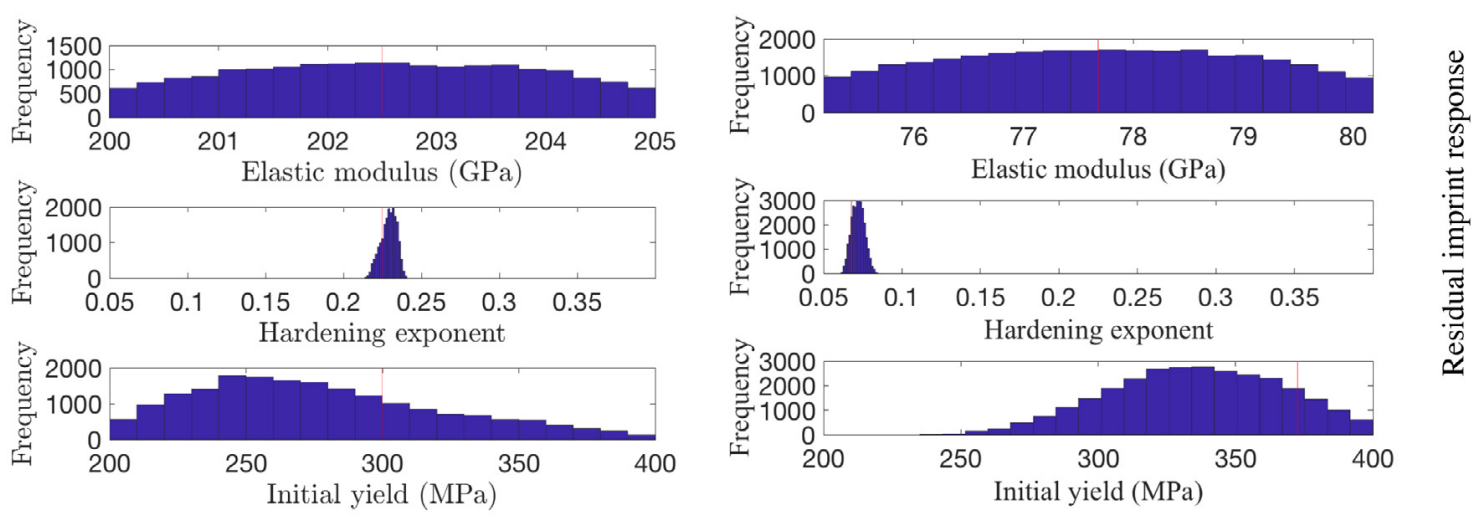

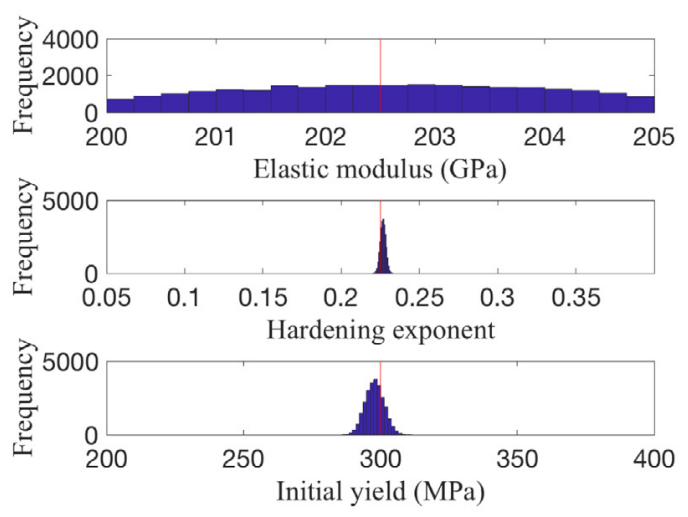

(a)

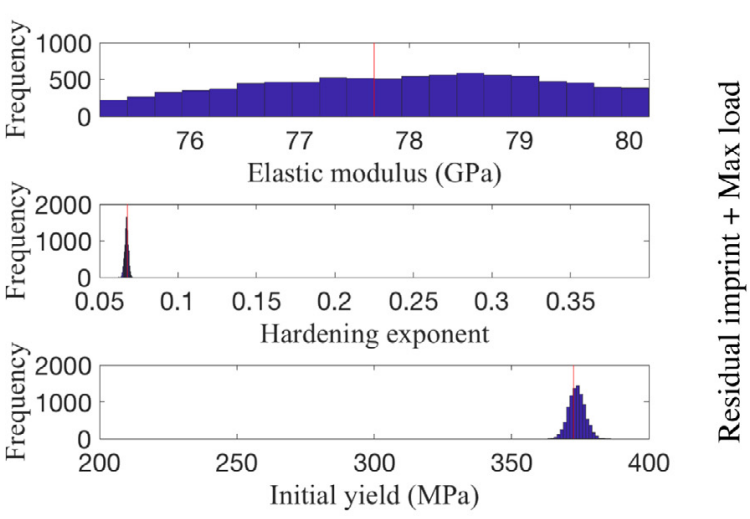

(b)

Fig. 4. Distributions of the identified parameters for model example 1, on the left, and model example 2, on the right. The system responses are imprint response for $0.17 \mathrm{~mm}$ indentation depth on top and the combination of imprint response and the maximum indentation load at the bottom.

For further exploration, we obtain the amount of information gain to infer each parameter independently. To this end, $p(\theta \mid D)$ in Eq. (6) is no longer the joint posterior distribution of the parameters, but is the posterior distribution of the initial yield or hardening exponent, marginalized on the rest of the parameters. The estimated amount of information gain to infer the parameters independently is represented in Table 6. As it is illustrated, considering maximum indentation load increases the information content of the material response to infer the model parameter. However, in comparison with the information content of the response to infer the initial yield, we noticed a sharp increase in the information content of hardening exponents. This is totally consistent with the observed posterior distributions in Fig. 4 and Table 6.

\subsubsection{Mutual information between the material response space and the material model space}

According to Eq. (5), the estimated information gain is a function of the observed material response. To extend our study, we apply the proposed framework to estimate the mutual information between the material response space and the material model space, with the latter represented by parameter ranges given in the first row of Table 3 . Such an estimate would represent the value of the "class" of the material response, rather than an observed response belonging to that class for an unknown material. Knowing the value of a particular material response class, we can then select the response class which contains the maximum information, on average, with respect to the material parameters. In addition, we can select the test's design parameters in such a way as to maximize the information content for a selected response.

Following the steps outlined in Section 2, we first estimate the mutual information between the space of the reaction force-indenter displacement response and the material model parameters while the maximum indenter depths are fixed at $0.17 \mathrm{~mm}$ and $0.44 \mathrm{~mm}$. We then estimate the mutual information while the material response is the combination of the residual imprint and the maximum indentation load. The results are represented in Table 7. As can be seen, 
Table 6

The amount of information obtained while inferring the marginalized model parameters.

\begin{tabular}{llll}
\hline Examples & Material response & Model parameter & Info. Gain estimated by nested sampling \\
\hline \multirow{3}{*}{ Model example 1 } & Residual Imprint & Hardening exponent & 2.83 \\
& Residual Imprint + Max load & Hardening exponent & 3.93 \\
& Residual Imprint & Initial yield & 0.16 \\
& Residual Imprint + Max load & Initial yield & 2.68 \\
\hline \multirow{3}{*}{ Model example 2 } & Residual Imprint & Hardening exponent & 3.09 \\
& Residual Imprint + Max load & Hardening exponent & 4.30 \\
& Residual Imprint & Initial yield & 0.52 \\
\hline
\end{tabular}

Table 7

Mutual information between the material model parameter space and the material response spaces.

\begin{tabular}{ll}
\hline Material response & Mutual information between response and parameter spaces \\
\hline Force displacement with the maximum indenter displacement $0.17 \mathrm{~mm}$ & 5.69 \\
Force displacement with indentation depth $0.44 \mathrm{~mm}$ & 8.2 \\
Imprint (indentation depth $=0.17)+$ Max load & 7.27 \\
Force displacement with indentation depth $=0.44 \mathrm{~mm}, \mathrm{C}=2 \mathrm{e}-3(\mathrm{~mm} / \mathrm{kN})$ & 7.75 \\
Force displacement with indentation depth $=0.44 \mathrm{~mm}, \mathrm{C}=4 \mathrm{e}-3(\mathrm{~mm} / \mathrm{kN})$ & 7.29 \\
\hline
\end{tabular}

the mutual information increases with the increase of the maximum indenter depth. When the material response is the combination of the residual imprint and the maximum indentation load, the value of the mutual information falls between that of the reaction force-indenter displacement for the maximum indenter depths $0.17 \mathrm{~mm}$ and $0.44 \mathrm{~mm}$. In other words, measuring the reaction force-indenter depth response while the maximum indenter depth is 0.44 is more informative than using the combination of residual imprint response and maximum load at a maximum depth of 0.17 .

Having explored the interplay between the material model parameter space and material response space we turn our attention the machine compliance uncertainty, which only affects the reaction force-indenter displacement. We introduce some uncertainty in the machine compliance as a test's "design parameter" and try to calibrate it such that the mutual information between the parameter space and the reaction force-indenter displacement response is equal to the mutual information when the combination of the imprint and maximum indentation load is used as response. The importance of identifying the uncertainty of the machine compliance is that we determine two different, yet equally worth-to-measure responses, one easy to measure, but involving more uncertainty sources, i.e. reaction force-displacement response and the other more difficult to measure requiring more sophisticated sensors, but independent of the knowledge of the machine compliance as the force measurement is independent of the displacement of the indenter. Minimizing the objective function defined as the difference between two mutual information values for two cases (the force-displacement as the response and the combination of the residual imprint and maximum indentation load as the response) the uncertainty of the machine compliance in which both responses are equally valued is determined. According to Table 7, when the upper bound for the range of machine compliance is equal to $4 \mathrm{e}-3(\mathrm{~mm} / \mathrm{kN})$, the mutual information between the response space and the parameter space for both cases is the same. We finally note that generating the objective function for each iteration of the optimization process is extremely demanding, as it requires estimating the mutual information for the selected machine compliance uncertainty. We therefore employed a Bayesian optimization technique [57] based on maximizing the expected improvement which for the particular case under consideration in this study was able to approach sufficiently close to target mutual information in a few iterations.

\section{Concluding remarks}

In this paper, we suggest posing the determination of material properties through inverse material parameter extraction in the context of information theory through considering the value of material responses, and taking due account of the sources of uncertainty.

We develop a computational framework which integrates Bayesian inference, nested sampling, finite element analysis and surrogate modeling through artificial neural network, where we exploit the efficiency of nested sampling 
in estimating the information gained by observing the material response and the mutual information between the material response space and the model parameter space.

We then apply the proposed framework to study the known responses of an indentation test, i.e. reaction force-indenter displacement, residual imprint and maximum indentation load responses, to infer a power law material hardening model. We illustrate how certain combinations of the material responses result in boosting the information content, also known as information fusion. We also show how the proposed framework can help design the experiment to maximize the information gain, or identify the alternative responses that are equally ranked from an information content perspective.

While we demonstrate the applicability of the proposed framework in the context of indentation tests, we expect it to be equally applicable to uncertainty-informed material identification in other experimental settings, e.g. punch test, bulge test, compression test, etc., as well as to design of experiments from the point of view of sensor selection and cost. It is in fact our expectation that such frameworks will assist experimental research, including the selection of the appropriate material tests, as they provide a quantitative basis for the examination of the trade-off between the cost associated with candidate tests and the value of their results.

\section{Acknowledgments}

This work was partially supported by the National Science Foundation (NSF), United States, Grants CMMI1235238 and CMMI-1351742. This support is gratefully acknowledged. The first and second authors would also like to acknowledge UP postdoctoral fellowship program and the Eskom Power Plant Engineering Institute (EPPEI) for their support.

\section{References}

[1] P.A. Prates, A.F.G. Pereira, N.A. Sakharova, M.C. Oliveira, J.V. Fernandes, Inverse strategies for identifying the parameters of constitutive laws of metal sheets, Adv. Mater. Sci. Eng. 2016 (2016) http://dx.doi.org/10.1155/2016/4152963.

[2] E. Asaadi, P.S. Heyns, Flow stress identification of tubular materials using the progressive inverse identification method, Eng. Comput. (Swansea, Wales) 33 (2016) 1472-1489, http://dx.doi.org/10.1108/EC-08-2015-0219.

[3] O.A. Babaniyi, A.A. Oberai, P.E. Barbone, Direct error in constitutive equation formulation for plane stress inverse elasticity problem, Comput. Methods Appl. Mech. Engrg. 314 (2017) 3-18, http://dx.doi.org/10.1016/j.cma.2016.10.026.

[4] S.U. Hamim, R.P. Singh, Proper orthogonal decomposition-radial basis function surrogate model-based inverse analysis for identifying nonlinear burgers model parameters from nanoindentation data, Trans. ASME, J. Eng. Mater. Technol. 139 (2017) http://dx.doi.org/10.1115 $/ 1.4037022$.

[5] S. Lalitha Sridhar, Y. Mei, S. Goenezen, Improving the sensitivity to map nonlinear parameters for hyperelastic problems, Comput. Methods Appl. Mech. Engrg. 331 (2018) 474-491, http://dx.doi.org/10.1016/j.cma.2017.11.028.

[6] K. Murdock, C. Martin, W. Sun, Characterization of mechanical properties of pericardium tissue using planar biaxial tension and flexural deformation, J. Mech. Behav. Biomed. Mater. 77 (2018) 148-156, http://dx.doi.org/10.1016/j.jmbbm.2017.08.039.

[7] H. Tari, M.J. Dapino, Globally convergent nonlinear 3D inverse model for smart materials with Hessian-based optimization, Comput. Methods Appl. Mech. Engrg. 318 (2017) 864-881, http://dx.doi.org/10.1016/j.cma.2017.02.019.

[8] E. Asaadi, D.N. Wilke, P.S. Heyns, S. Kok, The use of direct inverse maps to solve material identification problems: pitfalls and solutions, Struct. Multidiscip. Optim. 55 (2017) 613-632, http://dx.doi.org/10.1007/s00158-016-1515-1.

[9] M. Wang, D. Dutta, K. Kim, J.C. Brigham, A computationally efficient approach for inverse material characterization combining Gappy POD with direct inversion, Comput. Methods Appl. Mech. Engrg. 286 (2015) 373-393, http://dx.doi.org/10.1016/j.cma.2015.01.001.

[10] E. Asaadi, P.S. Heyns, A computational framework for Bayesian inference in plasticity models characterisation, Comput. Methods Appl. Mech. Engrg. 321 (2017) 455-481, http://dx.doi.org/10.1016/j.cma.2017.04.017.

[11] C. Gogu, R. Haftka, R.L. Riche, J. Molimard, Effect of approximation fidelity on vibration-based elastic c onstants identification, Struct. Multidiscip. Optim. 42 (2010) 293-304, http://dx.doi.org/10.1007/s00158-010-0493-y.

[12] C. Gogu, W. Yin, R. Haftka, P. Ifju, J. Molimard, R. Le Riche, A. Vautrin, Bayesian identification of elastic constants in multi-directional laminate from moiré interferometry displacement fields, Exp. Mech. 53 (2013) 635-648, http://dx.doi.org/10.1007/s11340-012-9671-8.

[13] J. Chiachío, M. Chiachío, A. Saxena, S. Sankararaman, G. Rus, K. Goebel, Bayesian model selection and parameter estimation for fatigue damage progression models in composites, Int. J. Fatigue 70 (2015) 361-373, http://dx.doi.org/10.1016/j.ijfatigue.2014.08.003.

[14] F. Ibisoglu, M. Modarres, Probabilistic life models for steel structures subject to Creep-Fatigue damage, Int. J. Prognostics Health Manage. 6 (2015).

[15] P. Fernandez-Zelaia, V. Roshan Joseph, S.R. Kalidindi, S.N. Melkote, Estimating mechanical properties from spherical indentation using Bayesian approaches, Mater. Des. 147 (2018) 92-105, http://dx.doi.org/10.1016/j.matdes.2018.03.037.

[16] H.H. Meidani, J.B. Bedrov, D.R.M. Kirby, Calibration and ranking of coarse-grained models in molecular simulations using bayesian formalism, Int. J. Uncertain. Quantif. 7 (2017) 99-115, http://dx.doi.org/10.1615/Int.J.UncertaintyQuantification.2017013407.

[17] G. Bolzon, M. Talassi, An effective inverse analysis tool for parameter identification of anisotropic material models, Int. J. Mech. Sci. 77 (2013) 130-144, http://dx.doi.org/10.1016/j.ijmecsci.2013.09.009. 
[18] L. Meng, P. Breitkopf, G. Le Quilliec, An insight into the identifiability of material properties by instrumented indentation test using manifold approach based on P-h curve and imprint shape, Int. J. Solids Struct. 106 (2017) 13-26, http://dx.doi.org/10.1016/j.ijsolstr.2016.12.002.

[19] M. Wang, J. Wu, Y. Hui, Z. Zhang, X. Zhan, R. Guo, Identification of elastic-plastic properties of metal materials by using the residual imprint of spherical indentation, Mater. Sci. Eng. A 679 (2017) 143-154, http://dx.doi.org/10.1016/j.msea.2016.10.025.

[20] N. Souto, S. Thuillier, A. Andrade-Campos, Design of an indicator to characterize and classify mechanical tests for sheet metals, Int. J. Mech. Sci. 101-102 (2015) 252-271, http://dx.doi.org/10.1016/j.ijmecsci.2015.07.026.

[21] X. Huan, Y.M. Marzouk, Simulation-based optimal Bayesian experimental design for nonlinear systems, J. Comput. Phys. 232 (2013), 288-317, http://dx.doi.org/10.1016/j.jcp.2012.08.013.

[22] Q. Long, M. Scavino, R. Tempone, S. Wang, Fast estimation of expected information gains for Bayesian experimental designs based on Laplace approximations, Comput. Methods Appl. Mech. Engrg. 259 (2013) 24-39, http://dx.doi.org/10.1016/j.cma.2013.02.017.

[23] E.G. Ryan, C.C. Drovandi, A.N. Pettitt, Fully Bayesian experimental design for pharmacokinetic studies, Entropy 17 (2015) 1063-1089, htt p://dx.doi.org/10.3390/e17031063.

[24] J. Liepe, S. Filippi, M. Komorowski, M.P.H. Stumpf, Maximizing the Information Content of Experiments in Systems Biology, PLoS Comput. Biol. 9 (2013) e1002888.

[25] J. Skilling, Nested sampling for general Bayesian computation, Bayesian Anal. 1 (2006) 833-860, http://dx.doi.org/10.1214/06-BA127.

[26] C.E. Shannon, A mathematical theory of communication, Bell Syst. Tech. J. 27 (1948) 379-423.

[27] D.V. Lindley, On a measure of the information provided by an experiment, Ann. Math. Statist. 27 (1956) 986-1005, http://dx.doi.org/10.121 4/aoms/1177728069.

[28] K. Mosegaard, A. Tarantola, Probabilistic approach to inverse problems, in: International Handbook of Earthquake \& Engineering Seismology, Academic Press, 2002, pp. 237-268.

[29] E.G. Ryan, C.C. Drovandi, M.H. Thompson, A.N. Pettitt, Towards Bayesian experimental design for nonlinear models that require a large number of sampling times, Comput. Statist. Data Anal. 70 (2014) 45-60, http://dx.doi.org/10.1016/j.csda.2013.08.017.

[30] L. Zhu, T. Dasgupta, Q. Huang, A D-optimal design for estimation of parameters of an exponential-linear growth curve of nanostructures, Technometrics 56 (2014) 432-442, http://dx.doi.org/10.1080/00401706.2013.866600.

[31] E.G. Ryan, C.C. Drovandi, J.M. McGree, A.N. Pettitt, a review of modern computational algorithms for bayesian optimal design, Internat. Statist. Rev. 84 (2016) 128-154, http://dx.doi.org/10.1111/insr.12107.

[32] D. Blackwell, Equivalent comparisons of experiments, Ann. Math. Statist. 24 (1953) 265-272, http://dx.doi.org/10.1214/aoms/1177729032.[33]

K.J. Ryan, Estimating expected information gains for experimental designs with application to the random fatigue-limit model, J. Comput. Graph. Statist. 12 (2003) 585-603, http://dx.doi.org/10.1198/1061860032012.

[34] N. Friel, J. Wyse, Estimating the evidence - a review, Stat. Neerl. 66 (2012) 288-308, http://dx.doi.org/10.1111/j.1467-9574.2011.00515.x.

[35] F. Feroz, M.P. Hobson, M. Bridges, MultiNest: An efficient and robust Bayesian inference tool for cosmology and particle physics,

Mon. Not. R. Astron. Soc. 398 (2009) 1601-1614, http://dx.doi.org/10.1111/j.1365-2966.2009.14548.x.

[36] F. Feroz, M.P. Hobson, Multimodal nested sampling: An efficient and robust alternative to Markov Chain Monte Carlo methods for astronomical data analyses, Mon. Not. R. Astron. Soc. 384 (2008) 449-463, http://dx.doi.org/10.1111/j.1365-2966.2007.12353.x.

[37] Mukherjee Pia, Parkinson David, R.L. Andrew, A nested sampling algorithm for cosmological model selection, Astrophys. J. Lett. 638 (2006) L51.

[38] J.R. Shaw, M. Bridges, M.P. Hobson, Efficient Bayesian inference for multimodal problems in cosmology, Mon. Not. R. Astron. Soc. 378 (2007) 1365-1370.

[39] J. Skilling, Bayesian computation in big spaces - Nested sampling and Galilean Monte Carlo, in: AIP Conference Proceedings, 2012, pp. 145-156, http://dx.doi.org/10.1063/1.3703630.

[40] S. Pathak, S.R. Kalidindi, Spherical nanoindentation stress-strain curves, Mater. Sci. Eng. R 91 (2015) 1-36, http://dx.doi.org/10.1016/j.ms er.2015.02.001.

[41] L.G. Olson, R.D. Throne, An inverse problem approach to stiffness mapping for early detection of breast cancer, Inverse Prob. Sci. Eng. 21 (2013) 314-338, http://dx.doi.org/10.1080/17415977.2012.700710.

[42] A. Chakraborty, P. Eisenlohr, Evaluation of an inverse methodology for estimating constitutive parameters in face-centered cubic materials from single crystal indentations, Eur. J. Mech. A Solids 66 (2017) 114-124, http://dx.doi.org/10.1016/j.euromechsol.2017.06.012.

[43] L. Meng, B. Raghavan, O. Bartier, X. Hernot, G. Mauvoisin, P. Breitkopf, An objective meta-modeling approach for indentation-based material characterization, Mech. Mater. 107 (2017) 31-44, http://dx.doi.org/10.1016/j.mechmat.2017.01.011.

[44] L. Meng, P. Breitkopf, B. Raghavan, G. Mauvoisin, O. Bartier, X. Hernot, Identification of material properties u sing indentation test and shape manifold learning approach, Comput. Methods Appl. Mech. Engrg. 297 (2015) 239-257, http://dx.doi.org/10.1016/j.cma.2015.09.004.

[45] M. Kim, S. Bang, F. Rickhey, H. Lee, Correction of indentation load-depth curve based on elastic deformation of sharp indenter, Mech. Mater. 69 (2014) 146-158, http://dx.doi.org/10.1016/j.mechmat.2013.10.002.

[46] G. Sun, F. Xu, G. Li, X. Huang, Q. Li, Determination of mechanical properties of the weld line by combining micro-indentation with inverse modeling, Comput. Mater. Sci. 85 (2014) 347-362, http://dx.doi.org/10.1016/j.commatsci.2014.01.006.

[47] D. Lim, J.H. Lee, Y. Choi, H. Lee, A numerical approach to spherical indentation techniques for creep property evaluation, Trans. Korean Soc. Mech. Eng. A 37 (2013) 1229-1237, http://dx.doi.org/10.3795/KSME-A.2013.37.10.1229.

[48] C.K.S. Moy, M. Bocciarelli, S.P. Ringer, G. Ranzi, Identification of the material properties of Al 2024 alloy by means of inverse analysis and indentation tests, Mater. Sci. Eng. A 529 (2011) 119-130, http://dx.doi.org/10.1016/j.msea.2011.09.005.

[49] G. Rauchs, J. Bardon, Identification of elasto-viscoplastic material parameters by indentation testing and combined finite element modelling and numerical optimization, Finite Elem. Anal. Des. 47 (2011) 653-667.

[50] J.M. Collin, G. Mauvoisin, P. Pilvin, Materials characterization by instrumented indentation using two different approaches, Mater. Des. 31 (2010) 636-640, http://dx.doi.org/10.1016/j.matdes.2009.05.043. 
[51] N. Ogasawara, N. Chiba, X. Chen, A simple framework of spherical indentation for measuring elastoplastic properties, Mech. Mater. 41 (2009) 1025-1033, http://dx.doi.org/10.1016/j.mechmat.2009.04.010.

[52] M. Zhao, N. Ogasawara, N. Chiba, X. Chen, A new approach to measure the elastic-plastic properties of bulk materials using spherical indentation, Acta Mater. 54 (2006) 23-32, http://dx.doi.org/10.1016/j.actamat.2005.08.020.

[53] J.H. Lee, T. Kim, H. Lee, A study on robust indentation techniques to evaluate elastic-plastic properties of metals, Int. J. Solids Struct. 47 (2010) 647-664, http://dx.doi.org/10.1016/j.ijsolstr.2009.11.003.

[54] Y. Zhang, J.D. Hart, A. Needleman, Identification of plastic properties from conical indentation using a bayesian-type statistical approach, J. Appl. Mech. 86 (2018) 011002-011002-9.

[55] X. Chen, N. Ogasawara, M. Zhao, N. Chiba, On the uniqueness of measuring elastoplastic properties from indentation: The indistinguishable mystical materials, J. Mech. Phys. Solids 55 (2007) 1618-1660, http://dx.doi.org/10.1016/j.jmps.2007.01.010.

[56] E. Billur, B. Çetin, O. Music, C. Şimşir, K. Davut, A Potential Solution to Mystical Materials in Indentation Test, Procedia Eng. 207 (2017) 1952-1957, http://dx.doi.org/10.1016/j.proeng.2017.10.967.

[57] E. Brochu, V.M. Cora, N. de Freitas, A Tutorial on Bayesian Optimization of Expensive Cost Functions, with Application to Active User Modeling and Hierarchical Reinforcement Learning, 2012. 